\title{
Coronary Spasm as a Cause of Perioperative Myocardial Infarction
}

\author{
Yoshinori Tanimoto, M.D., Yasuo Matsuda, M.D.,* \\ Yurio Kobayashi, M.D., Hironori Ebihara, M.D., \\ Eiji Hyakuna, M.D., Kiyoshi Takashiba, M.D., \\ Yoshio Hamada, M.D., and Tetsuro Kawashima, M.D.
}

\section{Summary}

The case of a patient with severe left main trunk disease is presented, in which spasm of the right coronary artery resulted in intraoperative myocardial ischemia and perioperative myocardial infarction. It is suggested that coronary spasm may be a cause of perioperative myocardial infarction, and has to be considered in case of unknown etiology.

\section{Additional Indexing Words:}

Coronary spasm Perioperative myocardial infarction

\begin{abstract}
CORONARY artery bypass graft has been widely used in the treatment of coronary artery disease. The complication most frequently reported is perioperative myocardial infarction. The etiology of this has not, however, been clarified entirely.

We present a case of coronary spasm as a cause of perioperative myocardial infarction.
\end{abstract}

\section{Case Report}

A 47-year-old man was doing well until 1 week before hospital admission when he started to notice an oppressive sensation in the chest associated with dyspnea. The symptoms were progressive, occurring with minimal activity, and in one instance, awakening him from sleep in the early morning. Each attack was relieved either by rest or disappeared spontaneously within several minutes. The patient was referred to our hospital following an abnormal ex-

From the Department of Cardiovascular Surgery and Internal Medicine, Saiseikai Shimonoseki General Hospital, Shimonoseki, Yamaguchi and *the Department of Internal Medicine, Yamaguchi University School of Medicine, Ube, Yamaguchi.

Address for reprint: Yoshinori Tanimoto, M.D., Department of Cardiovascular Surgery, Saiseikai Shimonoseki General Hospital, 3-4-1 Kifune, Shimonoseki, Yamaguchi 751, Japan.

Received for publication June 20, 1983. 

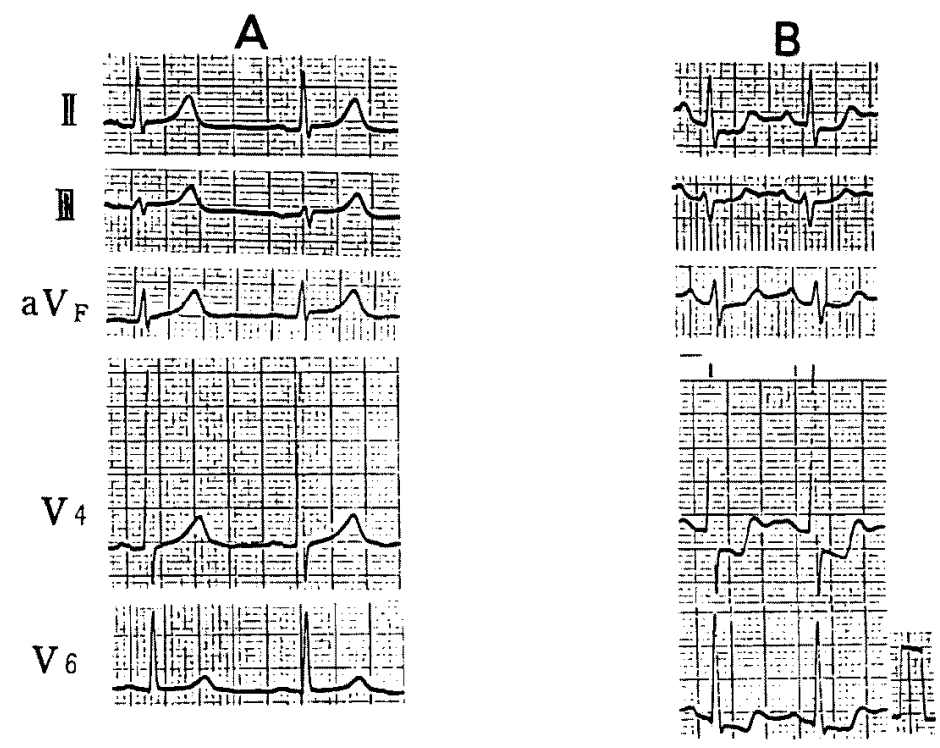

Fig. 1. Electrocardiograms at rest (A) and during exercise testing (B).

ercise test. He had a history of hypertension for 5 years and was smoking 1 to 2 packs of cigarettes per day for many years. His cholesterol level on admission was $259 \mathrm{mg} / \mathrm{dl}$. There was no history of diabetes. On admission, the blood pressure was $172 / 100 \mathrm{mmHg}$. The pulse rate was 60/min and regular. The remainder of the physical examination was unremarkable. Electrocardiograms at rest and during exercise are shown in Fig. 1. Following a Master's double test, the oppressive chest sensation developed, and ST-segment depression were noted in the left precordial leads. The chest X-ray was normal. Cardiac catheterization was performed during this hospitalization. Left ventricular pressure was $136 / 11 \mathrm{mmHg}$ and the left ventriculogram was normal. After administration of sublingual nitroglycerin, a fixed $90 \%$ stenosis of the left main coronary artery was observed. The left anterior descending coronary artery and left circumflex coronary arteries were normal. A mild narrowing of the proximal right coronary artery was observed (Fig. 2).

Soon after cardiac catheterization, a coronary artery bypass operation was performed under mild systemic hypothermia and cold cardioplegic arrest. The saphenous veins were grafted to the left anterior descending artery and the posterolateral branch of the circumflex artery. The patient came off bypass and was in regular sinus rhythm with good left ventricular contractility. While the chest was being closed, the patient developed a rapid visually apparent deterioration of right ventricular wall motion followed by ventricular premature beat and arterial hypotension. The cardiac monitor (lead II) showed apparent ST-segment elevation. This attack lasted a few minutes 


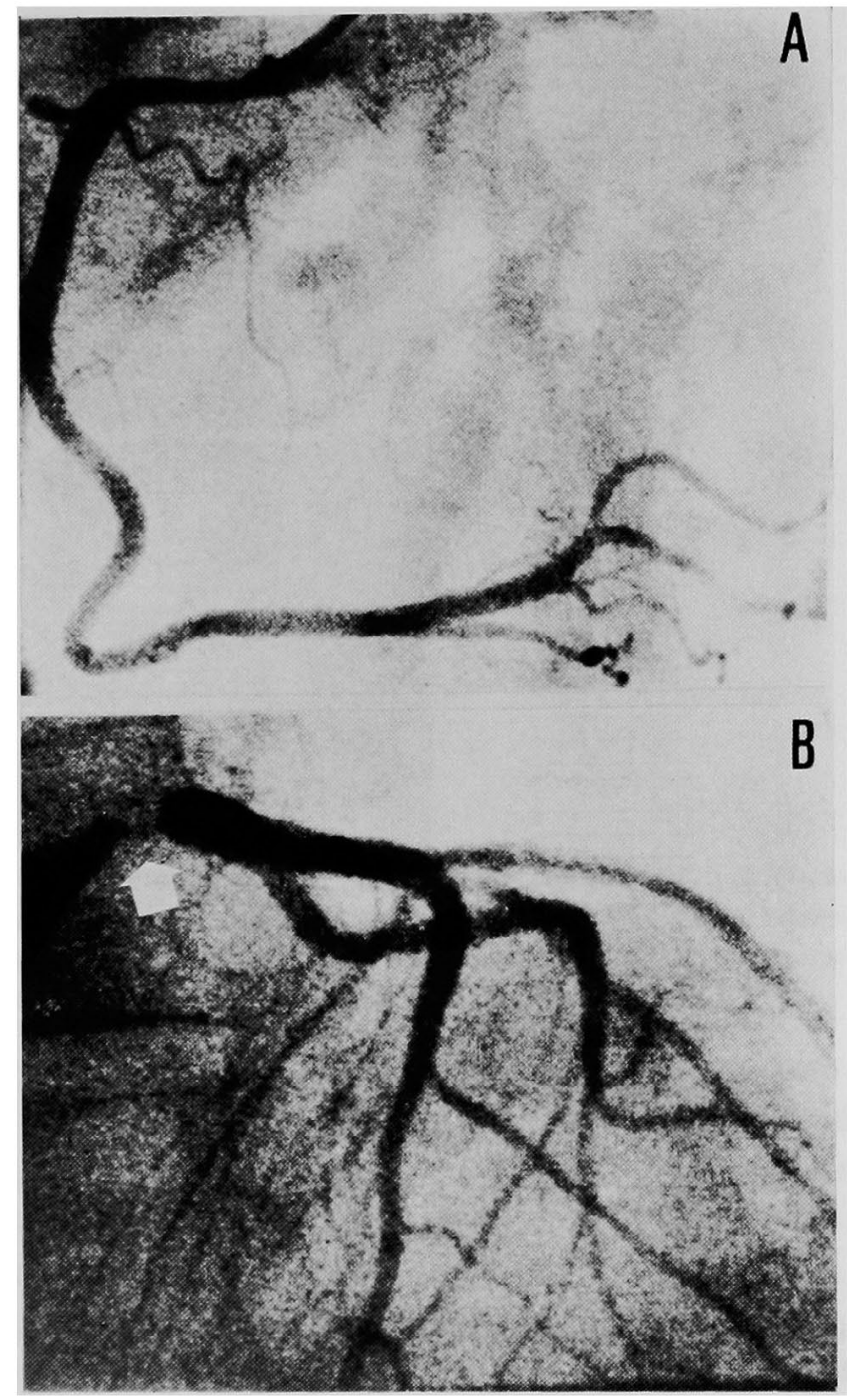

Fig. 2. Right coronary arteriogram in the left anterior oblique projection (A) and left coronary arteriogram in the anteroposterior projection (B). Severe organic stenosis of the left main coronary artery was observed (arrow).

and subsided spontaneously, following which it recurred every 15 to $20 \mathrm{~min}$. Intravenous nitroglycerin and $10 \mathrm{mg}$ of nifedipine administered via a nasogastric tube were not effective. During this period the chest was closed and the patient was transferred to the intensive care unit. After close of the chest, the patient's condition stabilized and no further attacks occurred. However, an electrocardiogram the following day showed $Q$ waves in leads II, III, and $\mathrm{aV}_{\mathrm{F}}$ (Fig. 3), and a significant increase of glutamic oxaloacetic transaminase to $134 \mathrm{IU}$, consistent with a perioperative inferior myocardial infarction. 

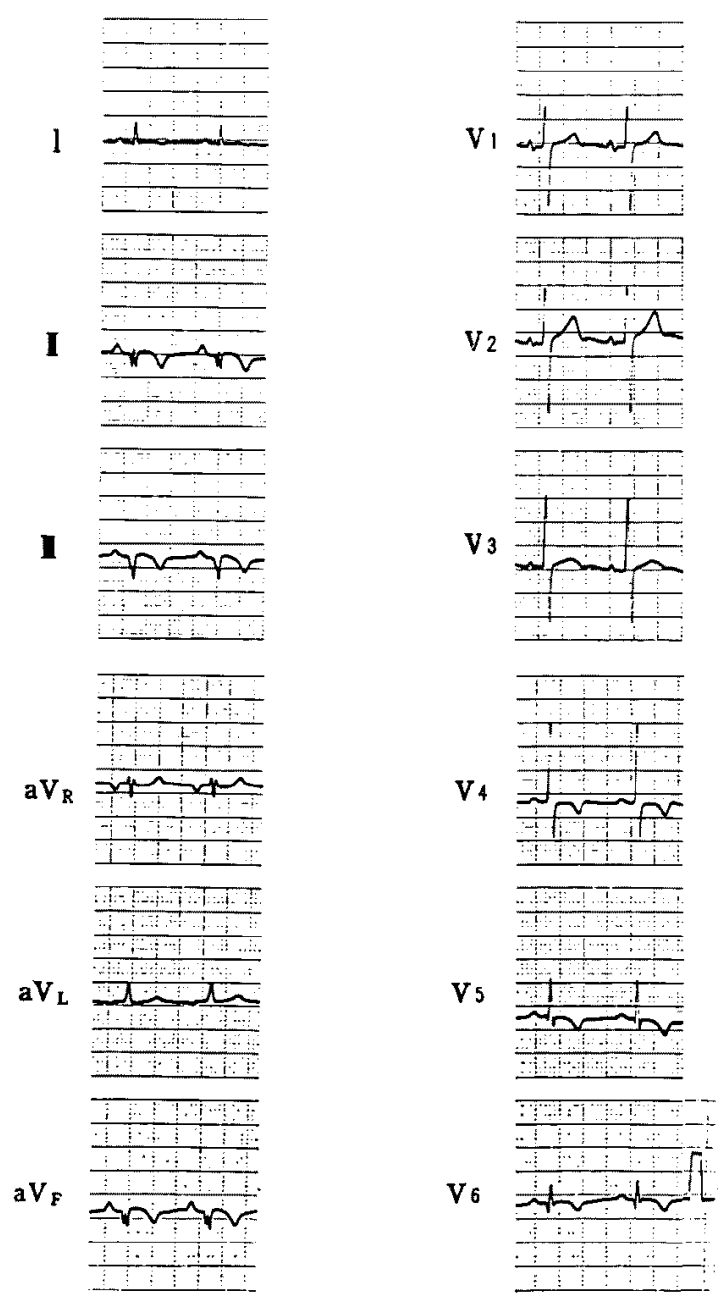

Fig. 3. Postoperative electrocardiogram. A new $Q$ wave was noted in leads II, III, and $\mathrm{aV}_{\mathrm{F}}$.

Three weeks following the bypass operation, cardiac catheterization was repeated. The left ventriculogram showed no significant impairment of the diaphragmatic wall. All grafts were patent. The appearance of the coronary arteries after administration of sublingual nitroglycerin was unchanged from the preoperative coronary arteriograms. A bolus of ergonovine maleate $(0.1 \mathrm{mg})$ was administered in the aortic root. Three min after the injection, the ST-segment elevation was seen in the inferior leads (Fig. 4), in association with anginal pain. Soon after this, the patient developed sinus bradycardia and arterial hypotension. Injection of contrast media into the right coronary artery revealed severe diffuse spasm with total occlusion in the midportion (Fig. 4-A). One half $\mathrm{mg}$ of atropine was administered intrave- 


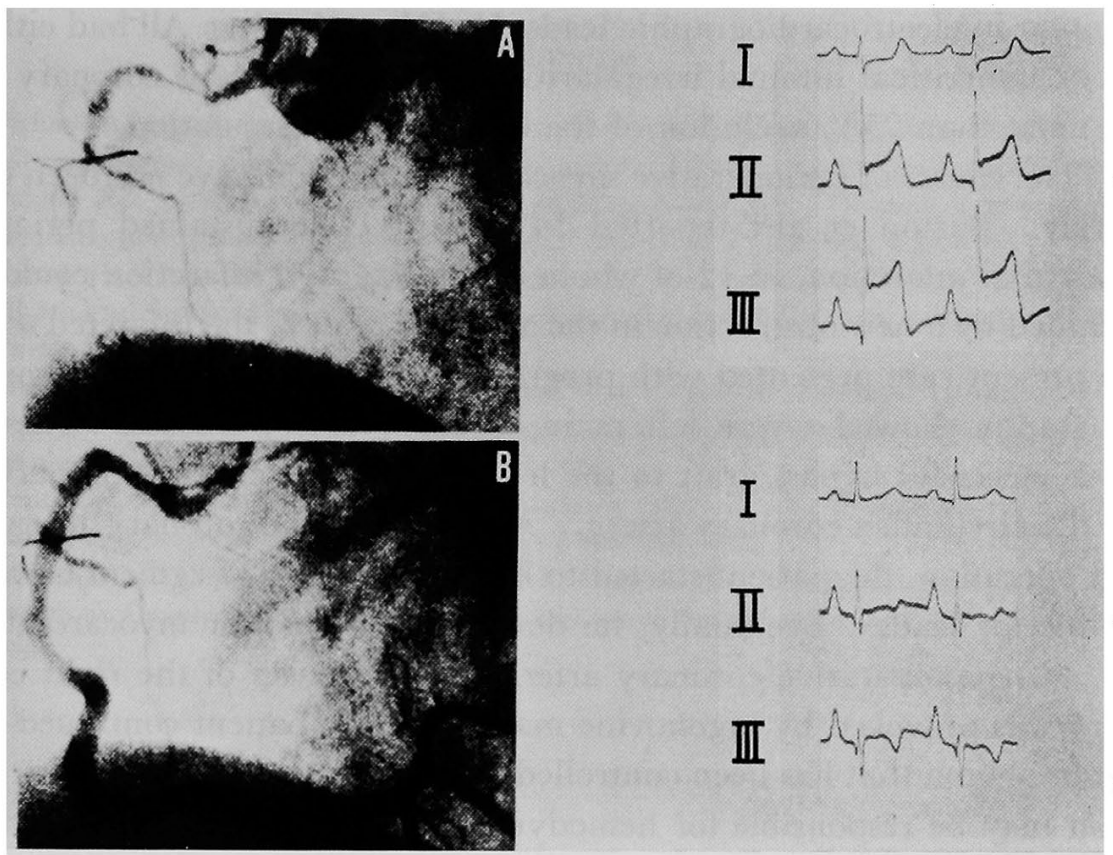

Fig. 4. Right coronary arteriograms (left panels) and electrocardiograms (right panels) during an anginal attack provoked by the administration of ergonovine maleate (A) and after the relief of angina by an intracoronary nitroglycerin (B).

nously, and $1 \mathrm{mg}$ of nitroglycerin was injected selectively into the right coronary artery following which the patient's symptoms subsided. Repeated opacification of the right coronary artery showed complete resolution of spasm (Fig. 4-B).

The patient was discharged on nifedipine $10 \mathrm{mg} 4$ times a day. Without medication, the patient has experienced attacks of angina at rest, especially in the morning or during the night.

\section{Discussion}

There has been increasing interest in the phenomenon of coronary artery spasm and its relationship to many clinical conditions related to myocardial ischemia. The occurrence of coronary artery spasm during or immediately after coronary bypass graft surgery has been reported..$^{1,2)}$ Pichard et a ${ }^{1)}$ reported a case with documented organic coronary artery disease in which coronary artery spasm was the probable cause of intraoperative cardiac arrest. Buxton et $\mathrm{al}^{2)}$ presented 6 patients who had unexpected hemodynamic collapse within 2 hours after cardiopulmonary bypass for myocardial revascularization. All 6 patients had profound hypotension and recurrent ST-segment 
elevation in electrocardiographic leads, II, III, and $\mathrm{aV}_{\mathrm{F}}$. All had either normal or noncritical luminal irregularities of dominant right coronary arteries and more than $75 \%$ occlusion of the left coronary circulation.

The causes of perioperative myocardial infarction have not been clarified entirely. Burton et al $^{3)}$ reported 54 patients who sustained perioperative myocardial infarction, in 12 of whom the myocardial infarction could not be explained by a new obstruction in the artery or graft to the infarcted segments. The present case presented with progressive anginal attacks and coronary arteriography showed severe left main coronary artery disease. The patient had a successful bypass graft to the left anterior descending coronary artery and the circumflex coronary artery. At the end of the coronary artery bypass graft operation, the patient started to have recurrent ST-segment elevation in the inferior leads. Eventually, he developed an inferior myocardial infarction. In postoperative coronary arteriography, spasm of the right coronary artery was provoked by ergonovine maleate. The patient continued to have variant angina that has been controlled by nifedipine. Thus, coronary artery spasm may be responsible for hemodynamic deterioration during and immediately after surgery, and one of the causes of perioperative myocardial infarction.

In the present case, coronary artery spasm occurred in a vessel other than the one with organic stenosis. Angina due to right coronary artery spasm might have been masked by his unstable angina due to organic left main trunk stenosis. Mercurio et al ${ }^{4)}$ reported 4 patients who had severe fixed coronary artery disease and in whom ergonovine mateate provoked spasm in the normal coronary artery. However, it has been reported that the use of ergonovine maleate in patients with severe fixed coronary artery disease has potential complications, especially in those patients with left main trunk disease. ${ }^{5)}$ Waters et al $^{6}$ ) reported 6 patients with significant organic coronary artery disease who underwent coronary artery bypass surgery. After surgery, rest angina with transient ST-segment elevation appeared in these patients in whom variant angina had not been previously diagnosed. The ergonovine maleate test may be valuable in those patients suspected of having had coronary artery spasm during and immediately after surgery, and those who continue to have angina pectoris postoperatively despite complete revascularization. In the future, a safe method should be developed to detect prior to surgery those patients with severe fixed coronary artery stenosis who are also prone to coronary spasm. Moreover, the prevention and prompt management of unexpected coronary artery spasm during surgery should be instituted where indicated. 


\section{REFERENCES}

1. Pichard AD, Ambrose J, Mindich B, Midwall J, Gorlin R, Litwak RS, Herman MW: Coronary artery spasm and perioperative cardiac arrest. J Thorac Cardiovasc Surg 80: 249, 1980

2. Buxton AE, Goldberg S, Harken A, Hirshfeld J, Kastor JA: Coronary-artery spasm immediately after myocardial revascularization. New Engl J Med 304: 1249, 1981

3. Burton JR, FitzGibbon GM, Keon WJ, Leach AJ: Perioperative myocardial infarction complicating coronary bypass. Clinical and angiographic correlations and prognosis. J Thorac Cardiovasc Surg 82: 758, 1981

4. Mercurio P, Kronzon I, Winer H: Spasm of a normal or minimally narrowed coronary artery in the presence of severe fixed stenoses of the remaining vessels. Clinical and angiographic observations. Circulation 65: 825, 1982

5. Conti CR, Feldman RL, Pepine CJ: Coronary artery spasm. Prevalence, clinical significance, and provocative testing. Am Heart J 103: 584, 1982

6. Waters DD, Theroux P, Crittin J, Dauwe F, Mizgala HF: Previously undiagnosed variant angina as a cause of chest pain after coronary artery bypass surgery. Circulation 61: 1159, 1980 